\title{
ON A PROPERTY OF THE BOUNDARY COR- RESPONDENCE UNDER QUASICONFORMAL MAPPINGS
}

\author{
KAZUO IKOMA
}

Let $w=f(z)$ be a quasiconformal mapping, in the sense of Pfluger [5]. Ahlfors [1], with maximal dilatation $K$, which will be simply referred to a $K-Q C$ mapping. As is well known, any $K-Q C$ mapping $w=f(z)$ of $\operatorname{Im} z>0$ onto $\operatorname{Im} w>0$ can be extended to a homeomorphism from $\operatorname{Im} z \geqq 0$ onto $\operatorname{Im}$ $w \geqq 0$ and hence it transforms any set of logarithmic capacity zero on $\operatorname{Im} z=0$ into a set with the same property on $\operatorname{Im} w=0$.

According to Beurling-Ahlfors [2], there exist a set $E$ of linear measure zero on $\operatorname{Im} z=0$ and a $K$-QC mapping $w=f(z)$ of $\operatorname{Im} z>0$ onto $\operatorname{Im} w>0$ such that the image set $f(E)$ of $E$ under $w=f(z)$ is of positive linear measure.

The purpose of this note is to prove the following theorem which is of some interest in contrast with the above theorem of Beurling-Ahlfors.

THEOREM. There exists on the real axis a closed set $E$ which is of linear measure zero and of positive logarithmic capacity and whose image set $f(E)$ under any K.QC mapping $w=f(z)$ of $\operatorname{Im} z>0$ onto $\operatorname{Im} w>0$ is of linear measure zero.

1. Take a closed segment $S_{1}$ with length $l_{1}$ on the real axis and delete from $S_{1}$ an open segment $T_{1}$ with length $\frac{l_{1}}{p_{1}}\left(p_{1}>1\right)$ such that the set $S_{2}=S_{1}$ $-T_{1}$ consists of two closed segments $S_{2}^{(j)}(j=1,2)$ with equal length $l_{2}$. In general, we delete from the set $S_{m-1}$ open segments $T_{m-1}^{(i)}\left(j=1,2, \ldots, 2^{m-2}\right)$ such that each $T_{m-1}^{(j)}$ has length $\frac{l_{m-1}}{p_{m-1}}\left(p_{m-1}>1\right)$ and the set $S_{m}=S_{m-1}$ $-\bigcup_{j=1}^{2^{m-2}} T_{m-1}^{(j)}$ consists of closed segments $S_{m}^{(j)}\left(j=1,2, \ldots, 2^{m-1}\right)$ with equal length $l_{m}$. It is obvious that the total length of the set $S_{m}$ is $2^{m-1} l_{m}=2^{m-2} l_{m-1}$ $\left(1-\frac{1}{p_{m-1}}\right)=l_{1} \prod_{n=1}^{m-1}\left(1-\frac{1}{p_{n}}\right), S_{m} \subset S_{m-1}$ and $\bigcap_{m=1}^{\infty} S_{m}$ is a non-empty perfect closed 
set. We denote the Cantor set $\bigcap_{m=1}^{\infty} S_{m}$ by $E\left(p_{1}, p_{2}, \ldots\right)$.

If we put $p_{n}=\frac{e^{n}}{e^{n}-1}(>1), n=1,2, \ldots$, then it holds the following relations :

$$
\begin{aligned}
& \prod_{n=1}^{\infty}\left(1-\frac{1}{p_{n}}\right)=0, \\
& \sum_{n=1}^{\infty} \frac{\log \left\{\frac{1}{\left(\frac{1}{2^{n}}-\frac{1}{p_{n}}\right)}{ }^{n}\right.}{2^{n}}<\infty
\end{aligned}
$$$$
2 p_{n+1}>p_{n}-1 \text {, }
$$

because $\sum_{n=1}^{\infty} \frac{1}{p_{n}}=\sum_{n=1}^{\infty}\left(1-\frac{1}{e^{n}}\right)=\infty, \sum_{n=1}^{\infty}\left\{\log 1 /\left(1-\frac{1}{p_{n}}\right)\right\} / 2^{n}=\sum_{n=1}^{\infty} n / 2^{n}<\infty$ and $2 p_{n+1}-p_{n}+1=\left\{\left(2 e^{n}-3\right) e^{n+1}+1\right\} /\left(e^{n+1}-1\right)\left(e^{n}-1\right)>0$. Hence we can see that the Cantor set $E\left(p_{1}, p_{2}, \ldots\right)$, where $p_{n}=\frac{e^{n}}{e^{n}-1}$, is of linear measure zero by $(\alpha)$ and is of positive logarithmic capacity by $(\beta)$ (cf. R. Nevanlinna [4]).

Now, take the systems $R_{n}=\left\{R_{n}^{(j)}\right\}_{j=1}^{2^{n}}(n=1,2, \ldots)$ constructed by Kuroda [3], consisting of concentric circular annuli such that the interior and exterior circles $C_{n}^{(j)}$ and $\Gamma_{n}^{(j)}$ of $R_{n}^{(j)}$ have the center at the middle point of $S_{n+1}^{(j)}$, and $C_{n}^{(j)}$ has the radius $r_{n}=\frac{l_{n}}{4}\left(1+\frac{1}{p_{n+1}}\right)\left(1-\frac{1}{p_{n}}\right)$ and $\Gamma_{n}^{(j)}$ has the radius $\rho_{n}=\frac{l_{n}}{4}\left(1+\frac{1}{p_{n}}\right)$. Then, it can be verified by using the preceding relation $(\gamma)$ that the segment $S_{n+1}^{(j)}$ lies inside the interior circle $C_{n}^{(j)}$ of $R_{n}^{(j)}$ and the exterior circle $\Gamma_{n+1}^{(j)}$ of $R_{n+1}^{(j)}$ lies inside some one of the interior circles of $R_{n}$. Further, we obtain as to the modulus of $R_{n}^{(j)}$ that

$$
\begin{aligned}
\bmod R_{n}^{(j)} & =\log \frac{1+\frac{1}{p_{n}}}{\left(1+\frac{1}{p_{n+1}}\right)\left(1-\frac{1}{p_{n}}\right)} \\
& \geqq \log \frac{1}{2\left(1-\frac{1}{p_{n}}\right)}=n-\log 2,
\end{aligned}
$$

which is valid for $j=1,2, \ldots, 2^{n}$.

2. Let $w=f(z)$ be any $K-Q C$ mapping stated in our theorem. If we define $w=f(z)$ in $\operatorname{Im} z<0$ by $\overline{f(\bar{z})}$, then it is well known that $w=f(z)$ can be extended to a $K \cdot Q C$ mapping in the whole plane. In this case, we may as- 
sume without loss of generality that the point at $\infty$ corresponds to each other under $w=f(z)$. Let $E$ be the Cantor set $E\left(p_{1}, p_{2}, \ldots\right)$, where $p_{n}=\frac{e^{n}}{e^{n}-1}(n$ $=1, \dot{2}, \ldots)$, lying on $\operatorname{Im} z=0$ and let $R_{n}=\left\{R_{n}^{(j)}\right\}_{j=1}^{2^{n}}(n=1,2, \ldots)$ be the systems constructed in 1 . Denote by $\widetilde{E}$ and $\widetilde{R}_{n}=\left\{\widetilde{R}_{n}^{(j)}\right\}_{j=1}^{2 n}(n=1,2, \ldots)$ the images of $E$ and $R_{n}=\left\{R_{n}^{(j)}\right\}_{j=1}^{n}(n=1,2, \ldots)$ under $w=f(z)$ respectively. Then it is evident that $\widetilde{E}$ is also closed and that $\widetilde{R}_{n}(n=1,2, \ldots)$ are the systems of doubly connected domains separating $w=\infty$ from $\widetilde{E}$ in the domain obtained by excluding $\widetilde{E}$ from the whole $w$-plane. It is well known that

$$
\frac{1}{K} \bmod R_{n}^{(j)} \leqq \bmod \widetilde{R}_{n}^{(j)}\left(j=1,2, \ldots, 2^{n}\right) .
$$

Next, denote by $\widetilde{C}_{n}^{(j)}$ and $\widetilde{\Gamma}_{n}^{(j)}$ the images of $C_{n}^{(j)}$ and $\Gamma_{n}^{(j)}$ under $w=f(z)$. Then, $\widetilde{C}_{n}^{(j)}$ and $\widetilde{\Gamma}_{n}^{(j)}$ are the interior contour and the exterior contour bounding $\widetilde{R}_{n}^{(j)}$ and are both symmetric with respect to the real axis $\operatorname{Im} w=0$. Moreover, we denote by $\widetilde{r}_{n}^{(j)}$ the largest distance of the image point of the center of $R_{n}^{(j)}$ from the contour $\widetilde{C}_{n}^{(j)}$, and denote by $\widetilde{\rho}_{n}^{(j)}$ the smallest distance from the contour $\widetilde{\Gamma}_{n}^{(j)}$. Then, by Teichmüller's theorem [6], we have

$$
\bmod \widetilde{R}_{n}^{(j)} \leqq \log \Psi\left(\frac{\widehat{\rho}_{n}^{(j)}}{\widehat{r}_{n}^{(j)}}\right) \quad\left(j=1,2, \ldots, 2^{n}\right),
$$

where $\log \Psi(P)$ is the modulus of Teichmüller's extremal domain whose two complementary continua are $\{w ;-1 \leqq R e w \leqq 0, \operatorname{Im} w=0\}$ and $\{w ; P \leqq R e$ $w \leqq+\infty, \operatorname{Im} w=0\}$.

From the two relations stated above, we have

$$
\frac{1}{K} \bmod R_{n}^{(j)} \leqq \log \Psi\left(\frac{\tilde{\rho}_{n}^{(j)}}{\widetilde{r}_{n}^{(j)}}\right) \quad\left(j=1,2, \ldots, 2^{n}\right) .
$$

3. First, note that if $P \geqq 8+6 \sqrt{2}$, then $16 P+8 \leqq P^{2}$, and hence $\Psi(P)<P^{2}$ from Teichmüller's inequality $\Psi(P)<16 P+8$.

Now, put $\log \Psi(8+6 \sqrt{2})=m_{\jmath}$. For any $K(1 \leqq K<\infty)$, choose an integer $n_{K}$ which is not less that $K m_{0}+\log 2$. Then, for $n \geqq n_{K}$,

$$
m_{0} \leqq \frac{1}{K}(n-\log 2) \text {. }
$$

If we combine (3) with (1) and (2), then we have for $n \geqq n_{K}$,

$$
m_{0}=\log \Psi(8+6 \sqrt{2}) \leqq \log \Psi\left(\frac{\widetilde{\rho}_{n}^{(j)}}{\widetilde{r}_{n}^{(j)}}\right) \quad\left(j=1,2, \ldots, 2^{n}\right) .
$$


Since $\Psi(P)$ is an increasing function of $P$, it follows that $8+6 \sqrt{2} \leqq \tilde{\rho}_{n}^{(j)} / \tilde{r}_{n}^{(j)}$. Therefore, we obtain for $n \geqq n_{\kappa}$,

or

$$
m_{0} \leqq \log \Psi\left(\frac{\widetilde{\rho}_{n}^{(j)}}{\widetilde{r}^{(j)}}\right)<2 \log \frac{\tilde{\rho}_{n}^{(j)}}{\widetilde{r}_{n}^{(j)}},
$$

$$
\widetilde{r}_{n}^{(j)} e^{m_{0} / 2} \leqq \tilde{\rho}_{n}^{(j)} \quad\left(j=1,2, \ldots, 2^{n}\right) .
$$

Summing up these, we have, for $n \geqq n_{K}$,

$$
e^{m_{0} / 2} \leqq \widetilde{\rho}_{n} / \widetilde{r}_{n},
$$

where $\widetilde{\rho}_{n}=\sum_{j=1}^{2^{n}} \tilde{\rho}_{n}^{(j)}$ and $\widetilde{r}_{n}=\sum_{j=1}^{2^{n}} r_{n}^{(j)}$.

Further, by a geometric consideration it is not difficult to see that

$$
\tilde{\rho}_{n} \leqq \widetilde{r}_{n-1} .
$$

Consequently, we have, for $n \geqq n_{K}$,

so that

$$
e^{m_{0} / 2} \leqq \widetilde{r}_{n-1} / \widetilde{r}_{n},
$$

$$
\prod_{n=n_{K}}^{N} e^{m_{0} / 2} \leqq \prod_{n=n_{K}}^{N} \frac{\widetilde{r}_{n-1}}{\widetilde{\gamma}_{n}}
$$

or

$$
e^{m_{0} / 2\left(N-n_{K}+1\right)} \leqq \frac{\widetilde{r}_{n_{K}-1}}{\widetilde{r}_{N}}
$$

Thus we have $\lim _{x \rightarrow \infty} \widetilde{r}_{N}=0$. This shows that the image set $\widetilde{E}$ of $E$ is of linear measure zero, and hence the Cantor set $E=E\left(p_{1}, p_{2}, \ldots\right)$, where $p_{n}=\frac{e^{n}}{e^{n}-1}$, is a required example.

\section{REFERENCES}

[1] L. Ahlfors: On quasiconformal mappings, Journ. d'Anal. Math., 3 (1953/54), 1-58.

[2] A. Beurling.L. Ahlfors: The boundary correspondence under quasiconformal mapping, Acta Math. 96 (1956), 125-142.

[3] T. Kuroda: A criterion for a set to be of 1-dimensional measure zero, to appear in Jap. Journ. Math.

[4] R. Nevanlinna: Eindeutige analytische Funktionen, Springer Verlag, 2 Aufl. (1953).

[5] A. Pfluger: Quelques theorems sur une classe de fonctions pseudo-analytiques, C. R. 231 (1950), 1022-1023.

[6] O. Teichmüller: Untersuchungen über konforme und quasikonforme Abbildung, Deutsche Math. 3 (1938), 621-678.

Department of Mathematics

Yamagata University 